$E$ coli strains isolated in the United Kingdom are drug resistant and that resistance is transferable in more than $60 \%$ of these. The majority of autotransferring resistance plasmids identified were of the B and F compatibility groups. Group B plasmids are particularly common in shigellae and have been observed in strains of Shigella dysenteriae 1 that have caused epidemics in Central America ${ }^{8}$ and Asia. ${ }^{9}$ F-like resistance plasmids are common in salmonellae, and $E$ coli and plasmids of the $\mathrm{F}_{\mathrm{I}}$ me group have been observed in salmonella strains that have caused outbreaks of gastroenteritis in the Middle East, ${ }^{10}$ India, ${ }^{11}$ and southern Europe. ${ }^{12}$

These findings are a cause for concern not simply because they indicate that the choice of treatment for severe illnesses is limited but also because they suggest the existence of a large pool of drug-resistant organisms in the community. Multiple drug resistance may be transferred in vivo to other pathogenic organisms including those $E$ coli responsible for systemic infections such as neonatal meningitis. Further studies are in progress to determine the prevalence of drug resistance in $E$ coli strains isolated from cerebrospinal fluid.

\section{References}

1 Rowe B, Threlfall EJ. Multiple antimicrobial resistance in enteric pathogens. F Antimicrob Chemother $1981 ; 7: 1-3$.
2 Anderson ES, Threlfall EJ. The characterisation of plasmids in the enterobacteria. 7 Hyg 1974; 72:471-87.

${ }^{3}$ Haltalin KC, Markley AH, Woodman E. Agar plate dilution method for routine antibiotic susceptibility testing in a hospital laboratory. $A m \mathcal{F}$ Clin Pathol 1973;60:384-94.

4 Anderson ES, Lewis MJ. Characterisation of a transfer factor associated with drug resistance in Salmonella typhimurium. Nature 1965;208: 843-9.

${ }^{5}$ Anderson ES. A rapid screening test for transfer factors in drug-sensitive Enterobacteriaceae. Nature 1965;208:1016-7.

6 Willshaw GA, Threlfall EJ, Ward LR, Ashley AS, Rowe B. Plasmid studies of drug-resistant epidemic strains of Salmonella typhimurium belonging to phage types 204 and 193. F Antimicrob Chemother 1980;6:763-73.

7 Lambert HP. Antimicrobial agents in diarrhoeal disease. Clin Gastroenterol $1979 ; 8: 827-33$

${ }^{8}$ Grindley NDF, Grindley JN, Anderson ES. R factor compatibility groups. Molecular and General Genetics 1972;119:287-97.

${ }^{9}$ Frost JA, Rowe B, Vandepitte J, Threlfall EJ. Plasmid characterisation in the investigation of an epidemic caused by multiply resistant Shigella dysenteriae type 1 in Central Africa. Lancet 1981 ;ii:1074-6.

10 Anderson ES, Threlfall EJ, Carr JM, McConnell MM, Smith HR. Clonal distribution of resistance plasmid-carrying Salmonella typhimurium, mainly in the Middle East. $f$ Hyg 1977;79:425-48.

11 Rowe B, Frost JA, Threlfall EJ, Ward LR. Spread of a multiresistant clone of Salmonella typhimurium phage type 66/122 in South East Asia and the Middle East. Lancet 1980; :1070-1.

${ }^{12}$ McConnell MM, Smith HR, Leonardopoulos J, Anderson ES. The value of plasmid studies in the epidemiology of infections due to drugresistant Salmonella wien. 7 Infect Dis 1979;139:178-89.

(Accepted 29 fune 1982)

\title{
Reuse of haemodialysis equipment: convenience and cost effectiveness
}

\author{
H K BANESTER， R D DRISCOLL， R N GREENWOOD， W R CATTELL
}

\begin{abstract}
The shortage of resources for providing renal replacement makes it essential to reduce revenue costs wherever possible. Assuming that haemodialysis is likely to remain a mainstay of renal replacement, a policy of reusing the expensive disposable dialysers and blood lines could offer substantial cost savings. Such reuse has been shown to be safe and to maintain efficiency, but it must also take account of convenience to the patient, especially those dialysing at home. A survey of patients treated with home dialysis shows that the rate of reuse of the complete extracorporeal circuit was highest for those patients who had automated reuse systems.
\end{abstract}

\section{Introduction}

The treatment of patients with end-stage renal failure by haemodialysis has been highly successful. Nevertheless,

\footnotetext{
Department of Nephrology, St Bartholomew's and St Leonard's Hospitals, London

H K BANESTER, HND, assistant administrator R D DRISCOLL, BA, MLITT, home administrator R N GREENWOOD, MSC, MRCP, research registra W R CATTELL, MD, FRCP, consultant physician
}

haemodialysis is expensive and several hundred patients die each year in the United Kingdom because the financial resources to provide treatment are lacking. ${ }^{12}$ In the United States, where there has been a quite lavish allocation of federal resources for dialysis programmes, there is increasing pressure to contain or reduce costs. ${ }^{34}$ At the same time most units in the United Kingdom and elsewhere are moving away from the use of the bulky Kiil-type artificial kidneys towards the use of small disposable dialysers, which require less work from staff and patients and take up less space in the home. This means, however, that the dialyser and other disposable equipment become a major revenue cost.

Though considerable savings could be made by reusing these disposable items, this policy has, however, met with resistance from both clinicians and patients on the grounds of safety, efficiency, and convenience. The safety and efficiency of the procedure is now widely accepted both in Europe and in the USA, ${ }^{5}{ }^{6}$ but convenience to, and acceptance by, patients has received little attention. Since most patients in the United Kingdom dialyse at home, the success of any policy aimed at more economic use of materials depends on their co-operation. A recent survey of home dialysis practice in our unit has given us the opportunity to examine the extent to which our own policy of reuse is implemented in the home.

\section{Cost benefits of reuse}

Single use of a disposable dialyser is expensive, about $£ 1900$ a year. Reuse (in our case to a total of six times) dramatically reduces this cost by more than half (figure). It is less commonly recognised that the arterial and venous blood lines, which-with the dialyser-form the extracorporeal circuit, are also expensive. If the dialyser can be reused 


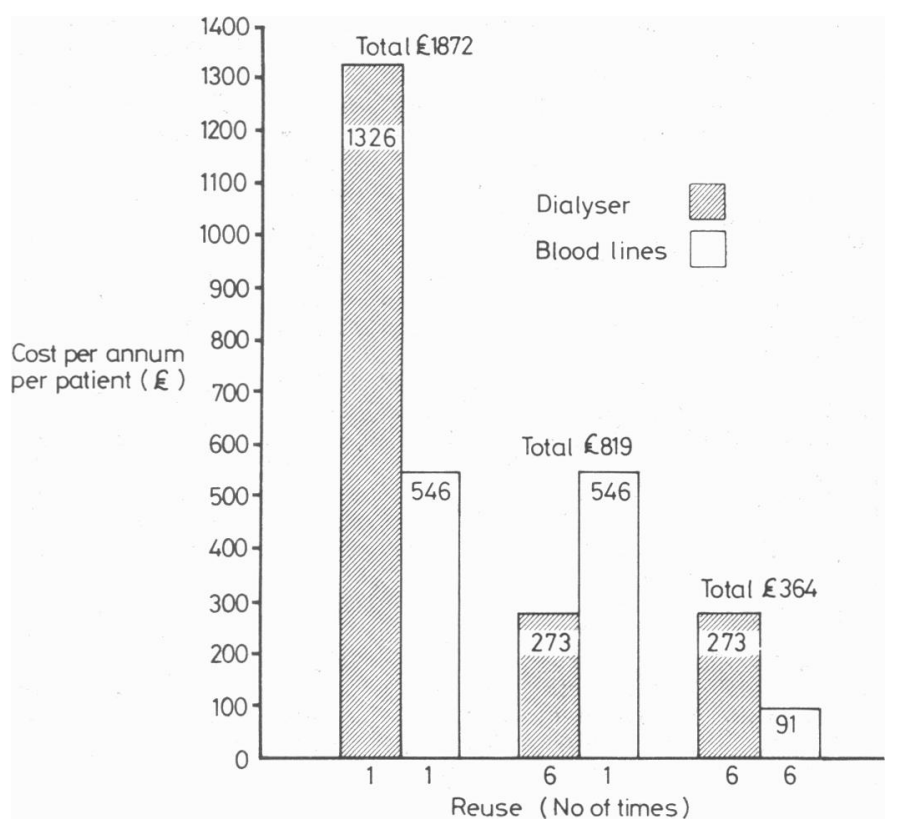

Costs per patient per year for use of dialysers and blood lines. (Allowance has been made for higher unit costs due to loss of bulk-purchase discounts.)

six times the cost of blood lines used only once constitutes $67 \%$ of the total cost of the extracorporeal circuit. Reusing blood lines to a maximum of six times can reduce costs still further. For a fair comparison of costs these must include not only the cost of major items but also that of materials used in reuse procedures: in our unit these amount to $£ 1.75$ per dialysis ( $£ 273$ a year). When added to the total amount this still shows a dramatic difference between single use $(£ 1872)$ and sixfold use of dialyser and lines ( $£ 637)$.

\section{Dialysis policy and practice}

On the basis of these financial considerations and evidence that reuse was safe and did not prejudice efficiency, ${ }^{7}{ }^{8}$ we decided to switch patients progressively to disposable dialysers, reusing both dialysers and lines a maximum of six times.

From a survey of patients dialysed at home giving details of the actual usage of dialysers and lines we were able to compare policy with practice. From a population of 72 patients on home haemodialysis, reliable data were obtained from $61(85 \%)$. Many of these (46) still used Kiil dialysers but 15 were using disposable dialysers; of these, six were operating a manual system for reuse while nine had automated systems using the Dylade DS equipment. Though the reuse of dialysers and lines varied considerably, certain important observations could be made.

Reuse of dialysers-On average Kiil dialysers were used 4.01 times without rebuilding (table). Such reuse has little economic value and must stem from convenience to the patient, as it is simpler to reuse than to rebuild a Kiil dialyser. Reuse of disposable dialysers occurred more frequently and reflects the patient's responsible attitude towards costs, since using a new dialyser each time would be more convenient. Reuse was marginally more frequent with the automated system.

Reuse of lines-For patients with Kiil dialysers the reuse rate was low (table); this may partly be because the patients were older and had

Cost of reusing dialysis equipment in practice. (Figures indicate mean No of times equipment reused)

\begin{tabular}{lcccc}
\hline & & \multicolumn{2}{c}{ Lines } & \multirow{2}{*}{$\begin{array}{c}\text { Annual cost } \\
\text { per patient }\end{array}$} \\
\cline { 3 - 5 } Equipment & Dialyser & Arterial & Venous & $£ 405^{*}$ \\
\hline Kiil & 4.01 & 1.37 & 1.57 & $£ 560$ \\
Disposable: & & 2.74 & 2.70 & $£ 448$ \\
$\begin{array}{l}\text { Manual reuse } \\
\text { Automated reuse }\end{array}$ & 4.64 & 5.00 & 5.00 & \\
\hline
\end{tabular}

* Capital cost of a Kiil dialyser has not been included. been trained initially to use dialyser and lines once only. The rate of reuse among patients on disposable dialysers was higher, reflecting both their training and an awareness of costs. The rate of reuse of the whole extracorporeal circuit where reuse procedures were automated was very high (table). The automated procedure is easier and less time consuming for patients to operate than the manual system, and these patients are clearly the most cost efficient.

\section{Discussion}

The unquestionable success of renal replacement programmes, whether by dialysis or transplantation for patients with end-stage renal failure, has created the extremely distressing situation in which many hundreds of patients now die in the United Kingdom each year because of lack of resources for treatment. Many factors are involved, but paramount among these is the lack of financial resources; in the present economic climate additional revenue is unlikely to be forthcoming. Indeed, far from expanding replacement programmes many centres find it difficult to sustain their present level of provision with presently available resources.

Given these hard facts, those concerned in this work need to examine the possible methods of achieving better provision from existing resources. Without question a considerable increase in successful cadaveric renal transplantation would be both more cost effective and more satisfactory to the patient. Sadly this has still yet to be achieved. Hopes that continuous ambulatory peritoneal dialysis might prove an equally successful but cheaper alternative to haemodialysis are at present threatened by the high incidence of recurrent peritoneal infections. For the foreseeable future regular haemodialysis must be an important mainstay of renal replacement. Assuming that self-supervised home dialysis must remain the principal method in the United Kingdom, we, like others throughout Europe and the United States, ${ }^{5}{ }^{9}$ believe that costs can be reduced by a policy of reusing disposable equipment. That dialysers can be reused without appreciable loss of efficiency has now been widely proved. ${ }^{5}{ }^{6}$ The safety of reusing dialysers has also been widely accepted. The cost benefit of reuse could be eliminated by cheaper manufacture of dialysers. The total cost of disposables must, however, take account of the cost of blood lines. Our own experience over several years has been that repeated use of blood lines is also safe; it not only reduces costs but is also more convenient for the patient if there is no need to connect and disconnect the lines for each dialysis. Cost-cutting exercises can only be effective if there is staff and patient compliance; this is especially important in home dialysis. This report suggests that this is most likely to be achieved by automated reuse procedures. The capital cost of equipment for automated reuse, especially if incorporated into automatic supply units, will be recouped in savings within the first year.

\section{References}

1 Office of Health Economics. Renal failure. A priority in health? London: OHE, 1978.

2 Jacobs C, Broyer M, Brunner FP, et al. Combined report on regular dialysis and transplantation in Europe, 1980: part 1. Proc Eur Dial Transplant Assoc 1982 (in press).

${ }^{3}$ Roberts SD, Maxwell DR, Gross TL. Cost-effective care of end-stage renal disease: a billion dollar question. Ann Intern Med 1980;92:243-8.

4 Lowrie EG, Hampers CL. Proprietary dialysis and the end-stage renal disease program. Dialysis and Transplantation 1982;11:191-204.

5 Vercellone A, Piccoli G, Alloatti S, et al. Reuse of dialyzers. Dialysis and Transplantation 1978;7:350-9.

6 Levin N. Dialyzer re-use in a hospital. Dialysis and Transplantation 1980;9:40-6.

7 Wing AJ, Brunner FP, Brynger HOA, et al. Mortality and morbidity of reusing dialysers. $\mathrm{Br} M e d \mathcal{F}$ 1978;ii:853-5.

${ }^{8}$ Miach PJ, Evans SM, Wilcox AA, Dawborn JK. Reuse of a disposable dialyser for home dialysis. Med $\mathcal{F}$ Aust $1976 ; 1: 146-7$.

${ }^{9}$ Ogden DA, Kopec G, Guy AD. Cost effectiveness of multiple dialyzer use. Dialysis and Transplantation 1981;10:407-11. 